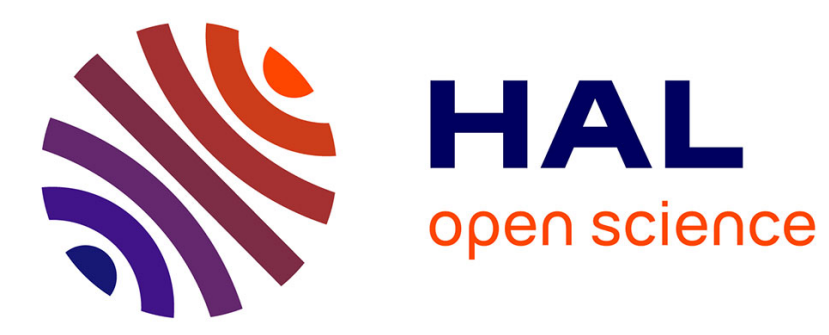

\title{
The cooperative dynamical behaviour of the ordered smectic phases
}

Michel Descamps, C. Descamps

\section{To cite this version:}

Michel Descamps, C. Descamps. The cooperative dynamical behaviour of the ordered smectic phases. Journal de Physique Lettres, 1984, 45 (9), pp.459-465. 10.1051/jphyslet:01984004509045900 . jpa00232369

\section{HAL Id: jpa-00232369 https://hal.science/jpa-00232369}

Submitted on 1 Jan 1984

HAL is a multi-disciplinary open access archive for the deposit and dissemination of scientific research documents, whether they are published or not. The documents may come from teaching and research institutions in France or abroad, or from public or private research centers.
L'archive ouverte pluridisciplinaire HAL, est destinée au dépôt et à la diffusion de documents scientifiques de niveau recherche, publiés ou non, émanant des établissements d'enseignement et de recherche français ou étrangers, des laboratoires publics ou privés. 
Classification

Physics Abstracts

$61.30 \mathrm{C}-64.70 \mathrm{M}$

\title{
The cooperative dynamical behaviour of the ordered smectic phases
}

\author{
M. Descamps \\ Laboratoire de Dynamique des Cristaux Moléculaires (*), \\ Université des Sciences et Techniques de Lille I, 59655 Villeneuve d'Ascq Cedex, France \\ and C. Descamps \\ Laboratoire des Molécules Diatomiques, \\ Université des Sciences et Techniques de Lille I, 59655 Villeneuve d'Ascq Cedex, France
}

(Reçu le 2 novembre 1983, révisé le 21 février 1984, accepté le 14 mars 1984)

\begin{abstract}
Résumé. - Dans cet article nous envisageons l'influence des répulsions stériques entre molécules d'un même plan smectique $G$ sur le comportement dynamique coopératif. Nous utilisons une approximation de champ moyen et un modèle de type Ising cinétique pour obtenir les susceptibilités dynamiques et l'expression de la diffusion neutronique cohérente $S(\mathbf{Q}, \omega)$. Les corrélations prises en compte induisent une dispersion des largeurs en $\omega$ qui est en accord avec les résultats expérimentaux de Levelut et al. sur T.B.B.A. Le modèle peut donner une estimation de l'influence des répulsions stériques sur le temps de relaxation incohérent.
\end{abstract}

\begin{abstract}
In this paper we investigate the influence of the steric repulsions between molecules within a smectic $G$ plane, on the cooperative dynamical behaviour. Within a mean-field theory of an extended kinetic Ising model, we derive dynamical equations which give the dynamical susceptibilities and the coherent neutron scattering function $S(\mathbf{Q}, \omega)$. The involved correlations induce a $\mathbf{Q}$ dispersion of the HWHM which is in accordance with the experiments of Levelut et al. on T.B.B.A. The model can lead to an estimate of the influence of the steric hindrance on the incoherent relaxation time.
\end{abstract}

At $113.5^{\circ} \mathrm{C}$ T.B.B.A. undergoes a transition from a crystalline phase to a smectic phase. In this phase the molecules are arranged in equidistant layers. Inside a layer they form a pseudohexagonal lattice, the distortion being due to the tilt of the molecules with regard to the normal to the layer. This phase is thus of $B_{c}$ type whose new, generally accepted, nomenclature [7] is smectic $G$. In this phase the molecules undergo orientational jump around their long axis. In X-ray experiments, performed on T.B.B.A., Levelut et al. [1] have shown the existence of diffuse zone boundary spots which reveal the existence of intermolecular correlations. The local order can be described by assuming the existence of geared local domains of three types; in each one the molecules arrange themselves in a herring-bone order. Four diffuse spots are associated

(*) E.R.A.-C.N.R.S. 465 . 
with each local cell. Thus, there appear 12 diffuse spots keeping the general pseudo-hexagonal symmetry. We have given an interpretation of the correlations based on a model taking into account the steric repulsion between phenyl rings [2]. The inelastic coherent neutron analysis of the diffuse zones of interest has been presented in $a^{x}$ recent paper by Levelut et al. [3]. We propose here a dynamic version of our model which we compare with these experiments. The aim is to show the influence of the reorientational hindrance on the $\mathbf{Q}$ dispersion of $\Gamma(\mathbf{Q})[$ the half width at half maximum of $S(\mathbf{Q}, \omega)]$. A short discussion of the influence of the correlations on the incoherent scattering is given at the end.

\section{The model and dynamic equations.}

We assume that the smectic phase is isotropic within the layer and that the molecules are at the sites of a plane hexagonal lattice. We take into account only the inplane molecular orientations belonging to the same steric configuration and we consider a model where the molecules are sterically symmetrical. The molecules assume three "steric » orientations which, for simplicity, are along the sides of the hexagonal lattice (Fig. 1). The molecules thus perform jumps into six positions. We assume that two neighbouring molecules within a plane would overlap with each other if they should get along the same side. Let us represent the orientation actually occupied by the $j$-th molecule by a three-component vector $\boldsymbol{\mu}^{j}$ such that :

$$
\begin{array}{ll}
\mu_{l}^{j}=1 & \text { if the molecular orientation at site } j \text { is actually } l \\
\mu_{l}^{j}=0 & \text { if not } .
\end{array}
$$

Considering that part of the interactions which relates to the hard core repulsions (quasi all-or-nothing interactions) we are statistically concerned with a compatibility function $A\left(S_{j} S_{i}\right)$ rather than the intermolecular potential. In the limit of complete restriction it takes either the value 1 or 0 depending of whether the molecular configurations $S_{i}$ and $S_{j}$ are compatible or not.

To take into account a possible softening of the repulsion it is suitable to write [4] :

$$
A\left(S_{j} S_{i}\right)=1-\lambda C_{j i}\left(S_{j}\right) C_{i j}\left(S_{i}\right) \quad(0<\lambda<1),
$$

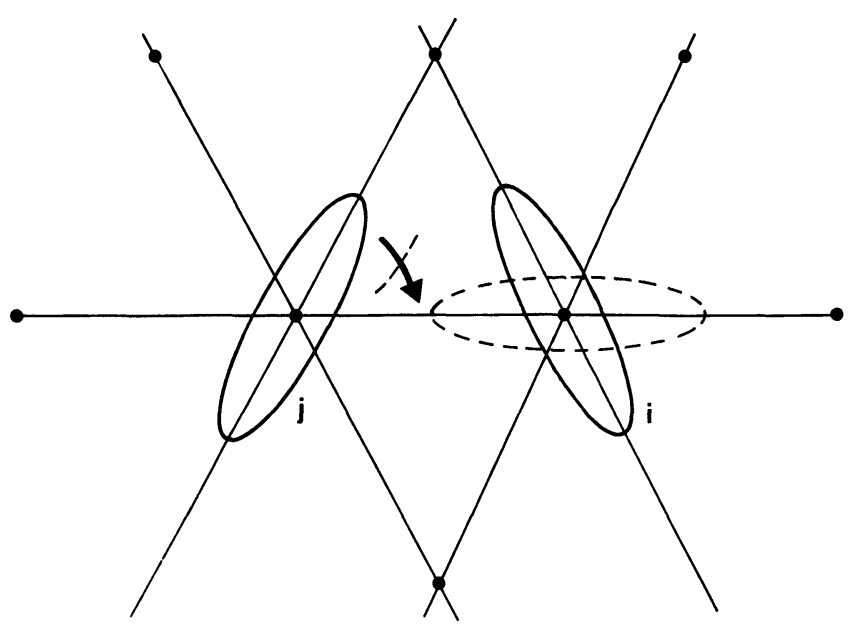

Fig. 1. - The model of disorder in the smectic B plane. The outline rotation of the $j$ molecule is impossible when it leads to steric contacts between the $j$ and $i$ molecules. 
where $C_{i j}\left(S_{i}\right)=1$ if the molecule $i$ in the state $S_{i}$ sterically fills the $i j$ edge

$$
=0 \text { otherwise } \text {. }
$$

The softening parameter $\lambda$ is related to the effective repulsive potential $W$ so that :

$$
\begin{aligned}
A\left(S_{j} S_{i}\right) & =1-\lambda=\mathrm{e}^{-\beta W} \quad \text { if } S_{i} \text { and } S_{j} \text { sterically interfere } \\
& =0 \text { otherwise. }
\end{aligned}
$$

In the mean field (M.F.) approximation, $A\left(S_{i} S_{j}\right)$ can be replaced by an effective interaction [4] :

$$
A_{j i}\left(S_{j}\right)=\left\langle A\left(S_{j} S_{i}\right)\right\rangle_{\langle i\rangle}=1-\lambda C_{j i}\left(S_{i}\right)\left\langle C_{i j}\left(S_{i}\right)\right\rangle_{\langle i\rangle}
$$

so that for example (Fig. 1) :

$$
A_{j i}\left(S_{j}=1\right)=1-\lambda\left\langle\mu_{1}^{i}\right\rangle ; \quad A_{j i}\left(S_{j} \neq 1\right)=1 .
$$

The dynamical description of the system of $N$ molecules requires the knowledge of the probability distribution $P\left(S_{1}, \ldots, S_{N} ; t\right)$ of the molecular system in the state $\left\{S_{1}, \ldots, S_{N}\right\}=\{S\}$ at time $t$. Its time dependence is assumed to be governed by a Markovian master equation :

$$
\begin{aligned}
\frac{\mathrm{d}}{\mathrm{d} t} P\left(S_{1}, \ldots,\left(S_{j}=l\right), \ldots, S_{N} ; t\right)= & -\sum_{j=1}^{N} \sum_{\substack{k=1 \\
\neq l}}^{3} \omega_{j}(l \rightarrow k) P\left(S_{1}, \ldots,\left(S_{j}=l\right), \ldots, S_{N} ; t\right) \\
& +\sum_{j=1}^{N} \sum_{\substack{k=1 \\
\neq l}}^{3} \omega_{j}(k \rightarrow l) P\left(S_{1}, \ldots,\left(S_{j}=k\right), \ldots, S_{N} ; t\right),
\end{aligned}
$$

where $\omega_{j}(l \rightarrow k)$ denotes the transition probability of the $j$-th molecule of going from state $l$ to state $k$. It must have a form which satisfies the detailed balance in equilibrium

$$
\frac{\omega_{j}(l \rightarrow k)}{\omega_{j}(k \rightarrow l)}=\frac{P_{0}\left(S_{1}, \ldots,\left(S_{j}=k\right), \ldots, S_{N}\right)}{P_{0}\left(S_{1}, \ldots,\left(S_{j}=l\right), \ldots, S_{N}\right)} .
$$

In the M.F. approximation we are thus led to choose [5]

$$
\omega_{j}(l \rightarrow k)=\frac{1}{\tau_{0}} \prod_{\langle j i\rangle}^{\mathrm{n} \cdot \mathrm{n}}\left\langle A_{j i}\left(k, S_{i}\right)\right\rangle_{i} \prod_{i}^{N} \prod_{l}^{3}\left(b_{l}^{i}\right)^{\mu_{i}\left(S_{i}\right)}
$$

where the first product runs over the sites sterically connected with $j . \tau_{0}$ denotes the relaxation time of a free molecule due to its interaction with a heat bath which is not treated explicitly. $b_{l}^{i}$ is a possible activity of the orientation $l$ at the site $i$. The correlation of the molecular motions is thus clearly related to the equilibrium statistics through the compatibility function.

Then the expectation value

$$
\left\langle\mu_{m}^{i}\right\rangle=\sum_{\{S\}} \mu_{m}^{i} P(\{S\} ; t)=\sum_{\{S\} \neq S_{i}} P\left(S_{1}, \ldots,\left(S_{i}=m\right), \ldots, S_{N} ; t\right)
$$

is found to obey a dynamical equation of the form

$$
\tau_{0} \frac{\mathrm{d}}{\mathrm{d} t}\left\langle\mu_{m}^{i}(t)\right\rangle=-\left\langle\mu_{m}^{i}(t)\right\rangle \sum_{k \neq m} \omega_{i}(m \rightarrow k)+\sum_{k \neq m}\left\langle\mu_{k}^{i}(t)\right\rangle \omega_{i}(k \rightarrow m) .
$$


Owing to (5) and (9) we get a set of consistency equations of the following form :

$$
\begin{aligned}
\tau_{0} \frac{\mathrm{d}}{\mathrm{d} t}\left\langle\mu_{1}^{0}\right\rangle=-\left\langle\mu_{1}^{0}\right\rangle\left\{\left(1-\lambda\left\langle\mu_{2}^{2}\right\rangle\right)\left(1-\lambda\left\langle\mu_{2}^{5}\right\rangle\right) b_{2}^{0}+\left(1-\lambda\left\langle\mu_{3}^{3}\right\rangle\right)\left(1-\lambda\left\langle\mu_{3}^{6}\right\rangle\right) b_{3}^{0}\right\}+ \\
+b_{1}^{0}\left\{\left(1-\lambda\left\langle\mu_{1}^{1}\right\rangle\right)\left(1-\lambda\left\langle\mu_{1}^{4}\right\rangle\right)\right\}\left[\left\langle\mu_{2}^{0}\right\rangle+\left\langle\mu_{3}^{0}\right\rangle\right] .
\end{aligned}
$$

In order to derive dynamical susceptibilities we linearize these equations with respect to small deviations $\delta\left\langle\boldsymbol{\mu}^{i}\right\rangle$ under the presence of oscillating external perturbations $\delta \mathbf{b}^{0} \exp (-i \omega t)$. Solving the system by Fourier transform and using the relation $\sum_{m=1}^{3} \delta\left\langle\mu_{m}(\mathbf{q}, t)\right\rangle=0$ we thus get the susceptibility matrix $\overline{\bar{\chi}}(\mathbf{q}, \omega)$ which is defined by

$$
\delta\left\langle\mu_{m}(\mathbf{q}, t)\right\rangle=\sum_{l} \chi_{m l}(\mathbf{q}, \omega) \delta b_{l}(\mathbf{q}) \exp (-i \omega t)
$$

\section{The neutron scattering cross section.}

The coherent scattering function is connected to the susceptibilities by [5]

$$
I_{\mathbf{c}}(\mathbf{Q}, \omega)=\operatorname{Tr}\left[\overline{\overline{\mathrm{F}}}^{\mathrm{T}}(\mathbf{Q}) \overline{\bar{\Phi}}(\mathbf{q}, \omega)\right] \quad(\mathrm{T}=\text { Transposition }),
$$

where $\overline{\overline{\mathbf{F}}}(\mathbf{Q})$ is a molecular form factor matrix.

$\overline{\bar{\Phi}}(\mathbf{q}, \omega)$ is the time Fourier transform of the relaxation function. In the particular case of a centred structure and centred molecules it reads

$$
\overline{\bar{\Phi}}(\mathbf{q}, \omega)=\frac{2 \overline{\bar{\chi}}^{\prime \prime}(\mathbf{q}, \omega)}{\omega} .
$$

By integrating over energy, the static susceptibility and the static intensity are obtained

$$
I_{\mathrm{c}}(\mathbf{Q})=\operatorname{Tr}[\overline{\overline{\mathrm{F}}}(\mathbf{Q}) \overline{\bar{\chi}}(q, \omega=0)] .
$$

2.1 Static COHERENT SCATTERING. - Levelut $e t$ al. [3] have measured the static scattering intensity $I_{\mathrm{c}}(\mathbf{Q})$. They have performed $\mathbf{Q}$ scans on the diffuse spots visible on X-ray diagrams. At $118.5^{\circ} \mathrm{C}$ the experimental full width at half maximum (FWHM) on the $[2,1,0]$ and $\left[\frac{5}{2}, \frac{1}{2}, 0\right]$ spots are $\Delta Q_{(\Delta)} \approx 0.5 \AA^{-1}$ in the $[3-\xi, \xi, 0]^{*}$ direction $(\Delta)$. The anisotropy is weak.

The present theory produces these spots (Fig. 2a) with the same localization of the maximum. The three zone boundary spots which appear on the figure are connected to the three possible local rectangular cell. Using an approximate Debye-Waller factor $\left(\left\langle u^{2}\right\rangle=0.3 \AA^{2}\right)$ the experimental FWHM in the $\Delta$ direction is found for $\lambda \approx 0.75$ corresponding to a mean repulsive energy of about $1.5 k T$ (Fig. 2b). The intensity in the valley [2.25, 1.75, 0$]$ is about $45 \%$ of the intensity of the spots.

2.2 INELASTIC COHERENT SCATTERING. - Under the assumptions included in our model we have calculated $I_{\mathrm{c}}(\mathbf{Q}, \omega)$ using the softening parameter $\lambda=0.75$ which correctly fits the experimental and theoretical static data at $118.5^{\circ} \mathrm{C}$. In each $\mathbf{Q}$ point, $I_{\mathrm{c}}(\mathbf{Q}, \omega)$ appears close to a Lorentzian. The present theory cannot give the absolute value of the line-width since the height of the potential barrier between the equilibrium orientations is not introduced. The results are presented taking as unity the microscopic time scale $\tau_{0}$ on which an uncorrelated reorientation takes place. We can get the $\mathbf{Q}$ dispersion rate of the $\operatorname{HWHM} \Gamma(\mathbf{Q})$. Curves are given along the $\Delta$ : $[3-\xi, \xi, 0]^{*}$ (Fig. 3a) and $[3 \xi, \xi, 0]^{*}$ (Fig. 3b)* directions. The maximum slowing down occurs 
(a)

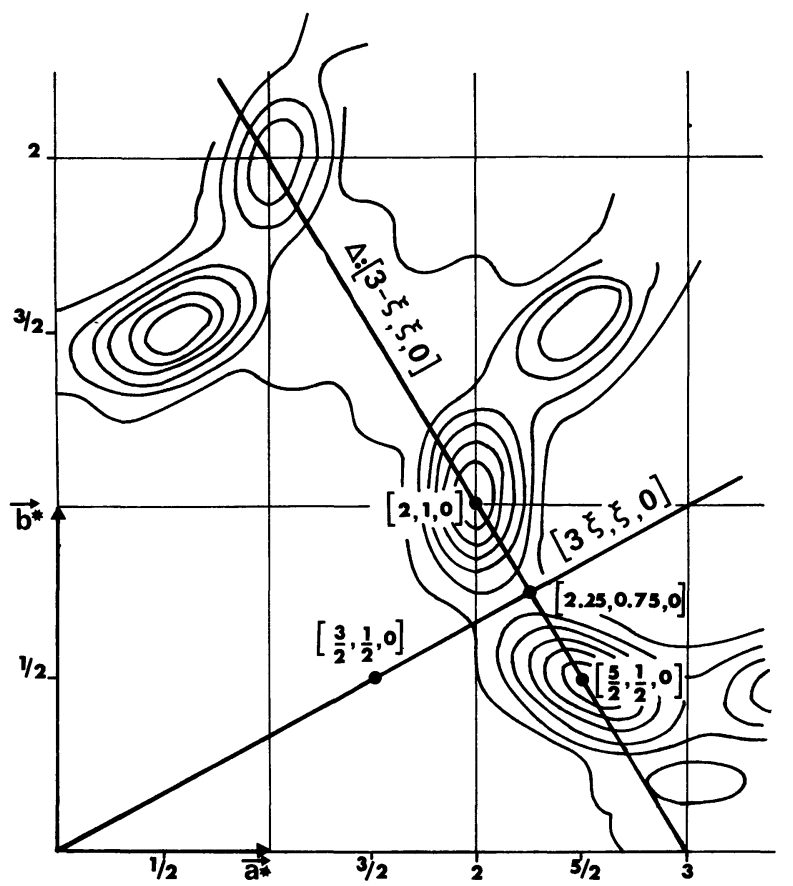

(b)

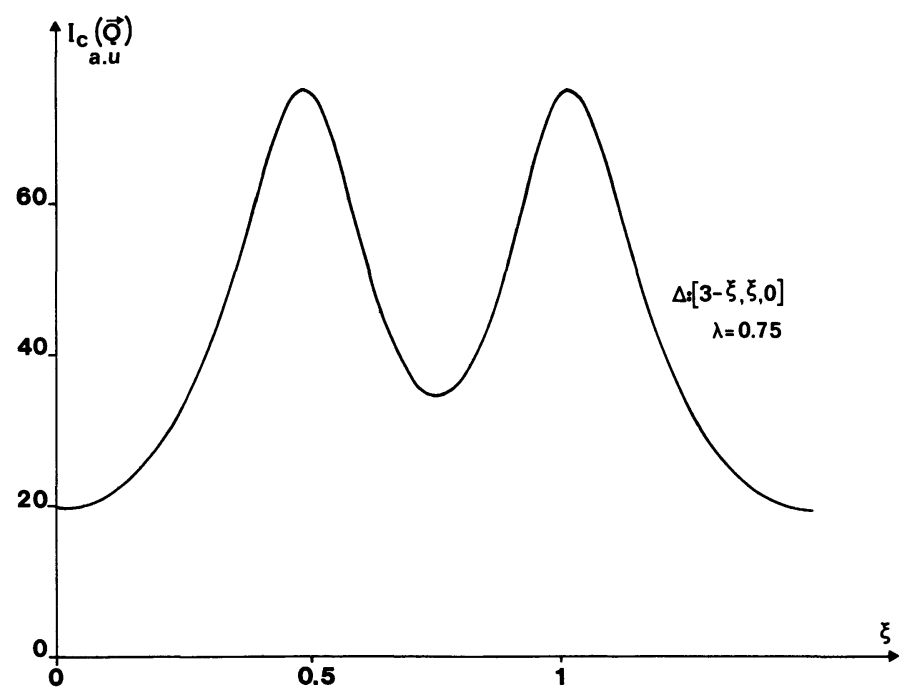

Fig. 2. - (a) Theoretical $I_{c}(\mathbf{Q})$ intensity map in the $\left(\mathbf{a}^{*}, \mathbf{b}^{*}\right)$ plane $(\lambda \approx 0.75)$. (b) Theoretical $\mathbf{Q}$ scan near the diffuse spots along the $\Delta:[3-\xi, \xi, 0]$ direction $(\lambda \approx 0.75)$.

in the spot region. The horizontal line at $\Gamma \simeq 8.3$ corresponds to the HWHM of an uncorrelated molecule. We can thus notice an " acceleration " at a distance of the spots. An inelastic broadening of $\frac{\Gamma(2.27,0.75,0)}{\Gamma(2,1,0)} \approx 1.6$ is found in the $\Delta$ direction for the points corresponding to the top and the valley of the static intensity. 
(a)
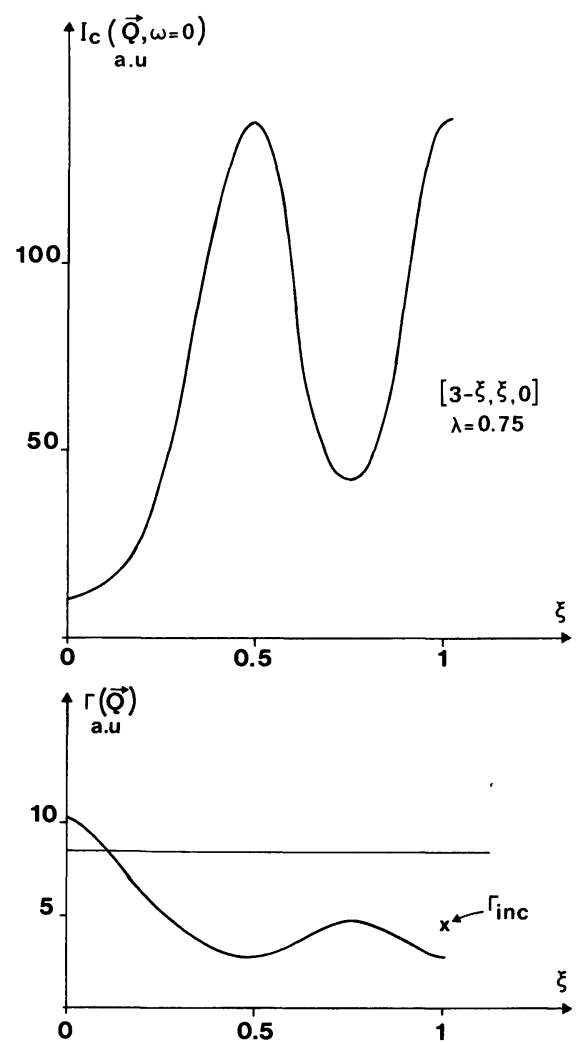

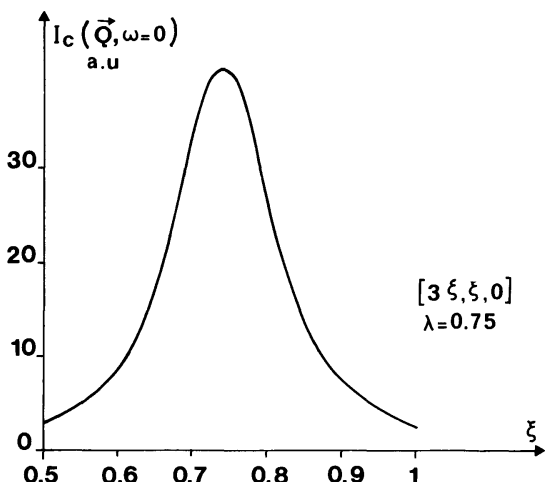

(b)

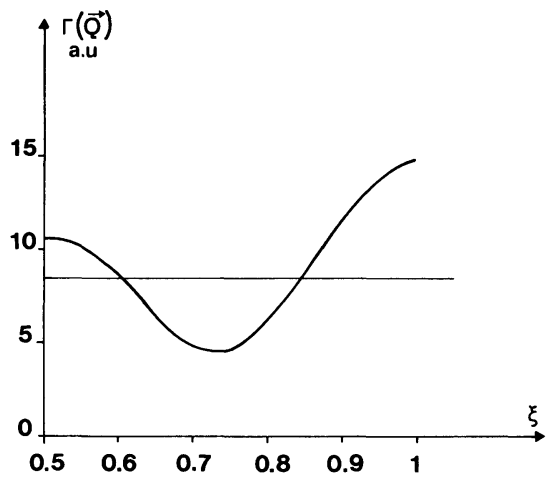

Fig. 3. - $\mathbf{Q}$ dispersion of $I_{\mathrm{c}}(\mathbf{Q}, \omega=0)$ (maximum of $I_{\mathrm{c}}(\mathbf{Q}, \omega)$ ) and $\mathbf{Q}$ dispersion of the FWHM $\Gamma(\mathbf{Q})$ (in unity $\tau_{0}=1$ ). (a) $\Delta:[3-\xi, \xi, 0]$ direction; (b) $[3 \xi, \xi, 0]$ direction. The horizontal line at $\Gamma \simeq 8$ corresponds to the FWHM of an uncorrelated molecule. The incoherent width $\Gamma_{\text {inc }}$ is pointed out at $[2,1,0]$ on Fig. 3a.

The $\mathbf{Q}$ dependence of the $I_{c}(\mathbf{Q}, \omega)$ maxima are depicted on figure 3 ; the following ratio has been found : $\frac{I(2.25,0.75,0 ; \omega=0)}{I(2,1,0 ; \omega=0)} \approx 0.3$.

The $\mathbf{Q}$ dependence observed by Levelut et al. at $111^{\circ} \mathrm{C}$ [3] do show the features predicted by the present calculation. The corresponding experimental broadening is 1.55 while the maximum ratio is 0.66 .

Thus the calculated contribution of the steric restriction to the dispersion rate of $\Gamma(\mathbf{Q})$ and $I_{c}(\mathbf{Q}, \omega=0)$ gives a correct quantitative interpretation of the experiments to within a factor of two. The accordance could be made better by introducing the in-plane anisotropy of the structure and refining the huge Debye-Waller factor. We should recall however that the static and dynamic experiments do not pertain to the same temperature.

2.3 QUASIELASTIC INCOHERENT SCATTERING. - In each of the three sterically different orientations of our model three are two sterically equivalent positions obtained by labelling the nuclei, so that the coherent scattering cannot distinguish $2 \pi / 3$ or $2 \pi / 6$ jump models. The labelling being necessary in the case of incoherent scattering, we can directly obtain the results of our model concerning the $2 \pi / 6$ jump model. Single molecule relaxation time $\tau_{\text {self }}$ is that of a single molecule experiencing reorientations correlated with its neighbours. It is directly deduced 
from (12) in term of $\tau_{0}$ (the relaxation time induced by the one thermal bath) by eliminating the site labelling :

$$
\tau_{\text {self }}^{-1}=(1-\lambda\langle\mu\rangle)^{2} \tau_{0}^{-1} \quad\left(\langle\mu\rangle=\frac{1}{3}\right)
$$

$\tau_{\text {self }}$ gives an idea of the global dynamic effect of the correlations. In the case of steric hindrance $(\lambda=1)$ the maximum augmentation is obtained : $\tau_{\text {self }}=\frac{9}{4} \tau_{0}$; for $\lambda=0.75$ we get $\tau_{\text {self }}=1.75 \tau_{0}$.

The HWHM of the corresponding incoherent function is pointed out at $[2,1,0]$ on figure $3 a$ in order to observe its position with respect to the different coherent widths.

The significant result is that :

$$
\Gamma\left(\begin{array}{lll}
2 & 1 & 0
\end{array}\right)<\Gamma_{\text {self }}<\Gamma\left(\begin{array}{lll}
1.5 & 0.5 & 0
\end{array}\right)
$$

which is in accordance with the experiments [6].

This result is consistent with the fact that in an incoherent experiment the correlations with other molecules are seen in a global way. This gives a width taking its value between those of maximum slowing down (2 10$)$ and maximum acceleration $\left(\begin{array}{llll}1.5 & 0.5 & 0\end{array}\right)$.

\section{Acknowledgments.}

We acknowledge several helpful conversations with Dr. A. M. Levelut.

\section{References}

[1] Levelut, A. M., J. Physique Colloq. 37 (1976) C3-411.

[2] Descamps, M., Coulon, G., Solid State Commun. 20 (1976) 379.

[3] Levelut, A. M., Moussa, F., Doucet, J., Benattar, J. J., Lambert, M., Dorner, B., J. Physique 42 (1981) 1651.

[4] DesCamps, M., J. Phys. C. 15 (1982) 7265.

[5] Descamps, M., J. Physique 45 (1984).

[6] Volino, F., Dianoux, A. J., Hervet, H., J. Physique Colloq. 37 (1976) C3-55.

[7] Gray, G. W., Philos. Trans. R. Soc. London, Ser. A 309, 77 (1983) 69-239. 